\title{
Association between positive urine cultures and necrotizing enterocolitis in a large cohort of hospitalized infants
}

Leslie C. Pineda ${ }^{\mathrm{a}}$, Christoph P. Hornik ${ }^{\mathrm{a}, \mathrm{b}}$, Patrick C. Seed ${ }^{\mathrm{a}}$, C. Michael Cotten ${ }^{\mathrm{a}}$, Matthew M. Laughon ${ }^{\mathrm{c}}$, Margarita Bidegain ${ }^{\mathrm{a}}$, Reese H. Clark ${ }^{\mathrm{d}}$, P. Brian Smith ${ }^{\mathrm{a}, \mathrm{b}}$

From the ${ }^{a}$ Department of Pediatrics, Duke University School of Medicine, Durham, NC, United States;

${ }^{b}$ Duke Clinical Research Institute, Duke University School of Medicine, Durham, NC, United States; ${ }^{c}$ Department of Pediatrics, The University of North Carolina at Chapel Hill, Chapel Hill, NC, United States; ${ }^{\mathrm{d}}$ Pediatrix-Obstetrix Center for Research and Education, Sunrise, FL, United States

Address for correspondence: P. Brian Smith, MD, MPH, MHS, Duke Clinical Research Institute, Box 17969, Durham, NC 27715; phone: 919-668-8951; fax: 919-668-7058; E-mail: brian.smith@ duke.edu.

Abbreviated Title: Association of Urinary Tract Infection with Necrotizing Enterocolitis 


\begin{abstract}
Objective: We used a large research database to examine the association between urinary tract infections and necrotizing enterocolitis (NEC) in premature infants.
\end{abstract}

Methods: This retrospective data analysis included infants $\leq 32$ weeks gestational age and $\leq 1500 \mathrm{~g}$ at birth who had urine cultures obtained at one of 322 neonatal intensive care units managed by the Pediatrix Medical Group from 1997-2012. The primary outcome was a diagnosis of NEC within 7 days after urine culture. We used multivariable conditional logistic regression conditioned on postnatal age and controlling for gestational age, inotropic support on the day of culture, and mechanical ventilation on the day of culture to evaluate the association between urine culture result and NEC.

Results: We identified 25,816 infants who had 43,556 urine cultures obtained; $6586(15.1 \%)$ of the cultures were positive. A diagnosis of NEC within 7 days after culture was made in $334(5.1 \%)$ of the 6586 positive cultures versus $1582(4.3 \%)$ of the 36,970 negative cultures $(\mathrm{p}<0.01)$. On multivariable analysis, infants with any positive urine culture had increased risk of NEC (odds ratio [OR] 1.16, 95\% confidence interval [CI] 1.02-1.31); the risk was higher when limited to Gram-negative organisms (OR $1.37,95 \%$ CI 1.17-1.59). The risk of surgical NEC was increased in infants with any positive urine culture (OR 1.46, 95\% CI 1.18-1.81) and was also higher when limited to Gram-negative organisms (OR $1.99,95 \%$ CI 1.53-2.59).

Conclusion: Positive urine cultures were associated with increased risk of NEC within 7 days of culture.

Key Words: neonatal, necrotizing enterocolitis, urinary tract infection 


\section{Abbreviations}

CoNS, coagulase-negative staphylococci

NEC, necrotizing enterocolitis

NICU, neonatal intensive care unit

UTIs, urinary tract infections

VLBW, very low birth weight 


\section{Introduction}

Necrotizing enterocolitis (NEC) occurs in $5 \%$ to $11 \%$ of very low birth weight (VLBW; <1500 g) infants [1,2]. NEC likely results from a combination of several factors, including genetic predisposition, intestinal immaturity, excessive intestinal inflammatory response, and inappropriate microbial colonization $[3,4]$. NEC mortality ranges from $16 \%$ to $42 \%$ [5]. Survivors are at high risk of severe growth delay and poor neurodevelopmental outcomes [6,7].

NEC has previously been associated with preceding gastrointestinal, respiratory, and bloodstream infections [8-10]. Outbreaks of NEC have been described, suggesting a transmissible infectious factor. Despite these reports, no single pathogen has been identified. A shift in the intestinal microbial ecology is thought to contribute to NEC risk in premature infants. This shift is preceded and marked by a loss of overall diversity and an increase in the proportion of bacterial families such as the Enterobacteriaceae [11-13]. Enterobacteriaceae includes Escherichia coli, Klebsiella spp., and Enterobacter spp., which are among the most commonly isolated organisms in urinary tract infections (UTIs) affecting infants in the neonatal intensive care unit (NICU) [14,15]. We examined the association between UTIs and NEC using a large research database.

\section{Methods}

\subsection{Study cohort}

We identified a cohort of all infants discharged from 322 NICUs managed by the Pediatrix Medical Group from 1997-2012. The data were obtained from an electronic medical record that prospectively captured data from admission notes, daily progress notes, procedure notes, and discharge summaries. Information was collected regarding maternal history and demographics, medications, laboratory results, culture results, and diagnoses.

We included all infants who were $\leq 32$ weeks gestational age and weighed $\leq 1500 \mathrm{~g}$ at birth and who had urine cultures obtained via supra-pubic tap, in-and-out catheterization, or bag in the first 120 days of life. We excluded infants whose only urine cultures were obtained after developing NEC, as well 
as those with congenital gastrointestinal or urogenital defects. All urine cultures obtained prior to a diagnosis of NEC or 120 days of life were included in the analysis (Figure). We included definite and probable episodes of coagulase-negative staphylococci (CoNS) infection as defined using previously reported criteria: a definite CoNS infection was defined as two positive cultures on the same day; a probable CoNS infection as two positive cultures within a 4-day period, three positive cultures within a 7day period, or four positive cultures within a 10-day period; and a possible CoNS infection as a culture positive for CoNS that did not meet criteria for definite or probable CoNS sepsis [16-18]. The diagnosis and severity of NEC were assigned at each site by the attending neonatologist and included either medical NEC or surgical NEC. NEC severity was defined on the first day of the course of NEC regardless of change in severity thereafter.

\subsection{Statistical analysis}

The unit of observation for this study was a urine culture. The primary outcome was a diagnosis of NEC occurring within 7 days after the urine culture was obtained. Infant-level categorical variables were compared between patients with and without NEC following the urine culture using chi-square tests and Wilcoxon rank sum tests. We used multivariable conditional logistic regression conditioned on postnatal age in days at the time of urine culture to evaluate the association between urine culture and NEC. In the final model, we included the following covariates: gestational age, inotropic support on the day of culture, and mechanical ventilation on the day of culture. All analyses were performed using Stata 12 (College Station, TX) and assumed a significance limit of $\alpha=0.05$. The study was approved by the Duke University Institutional Review Board without the need for written informed consent as the data were collected without identifiers.

\section{Results}

We identified 25,816 infants with 43,556 urine cultures. The median gestational age and birth weight were 27 weeks (interquartile range [IQR] 25-29) and 915 g (IQR 720-1154), respectively. Infants 
who developed NEC had a lower median birth weight compared to those who did not develop NEC (880 g, IQR 700-1106, vs. 920 g, IQR 725-1160, respectively; p<0.001) (Table 1).

There were 6586/43,556 (15.1\%) positive urine cultures in 5675 infants. Among those with positive urine cultures, the median postnatal age at the time of urine culture was 16 days (IQR 7-30) in those who did not develop NEC and 19 days (IQR 12-29) in those who developed NEC within 7 days. The organism most commonly cultured from the urine was Escherichia coli (1079/6586, 16.4\%), followed by Klebsiella $(940 / 6586,14.3 \%)$ and Enterococcus $(917 / 6586,13.9 \%)$. Among infants with positive urine cultures who subsequently developed NEC within 7 days $(\mathrm{N}=334)$, the most common organism cultured from the urine was Klebsiella $(62 / 334,18.6 \%)$ (Table 2). Infants with positive urine cultures who did not develop NEC ( $\mathrm{N}=6252)$ most commonly had urine cultures growing Escherichia coli $(1028 / 6252,16.4 \%)$. We identified 1916 cases of NEC within 7 days of a urine culture. NEC was diagnosed more frequently within 7 days of a positive culture than a negative culture (334/6586 [5.1\%] vs. $1582 / 36,970$ [4.3\%], $\mathrm{p}<0.01)$. Of the 334 NEC cases following a positive urine culture, $108(32.3 \%)$ were surgical NEC, and 226 (67.7\%) were medical NEC. Using multivariable analysis, odds of NEC were increased in infants with any positive urine culture (OR 1.16, 95\% CI 1.02-1.31), and the odds were higher when limited to urine cultures with Gram-negative organisms (OR 1.37, 95\% CI 1.17-1.59). Odds of surgical NEC were increased in infants with any positive urine culture (OR 1.46, 95\% CI 1.18-1.81), and the odds were higher when limited to urine cultures with Gram-negative organisms (OR 1.99, 95\% CI 1.53-2.59). We did not observe an association between NEC and Gram-positive urinary tract infections (OR 1.00, 95\% CI 0.80-1.27) (Table 3).

\section{Discussion}

We identified UTI as a risk factor in the development of NEC. An NEC diagnosis within 7 days was made more frequently after positive than negative urine cultures, although the absolute difference was small. Compared to infants with negative urine cultures, infants with positive urine cultures had an 
increased risk of NEC, especially surgical NEC, and the risk was higher when limited to Gram-negative organisms.

Although not specifically studied in the premature infant, colonization and infection of the urinary tract is generally regarded as an ascending process whereby organisms originate from the intestinal microbiota and ultimately colonize the peri-urethral region $[19,20]$. Thus urine cultures may serve as sentinels for intestinal dysbiosis, reflecting shifts in the intestinal and peri-urethral microbiota. Non-catheterized urine collections, in particular, may reflect colonization of the peri-urethral niche and thus best reflect changes in the intestinal microbiota. We were unable to differentiate between specimens collected via bag or in-and-out catheterization.

Although there are very limited data relating UTIs to gastrointestinal pathology in hospitalized infants [21], other serious infections have been linked with NEC. There are reports of NEC following infection as well as decreased incidence of NEC with infection control measures [22]. In a 1-month outbreak of 18 NEC cases, a preceding cluster of gastroenteritis cases was observed, but no particular causative organism identified [8]. Early colonization with Clostridium was also shown in 12 VLBW infants who later developed NEC [23]. Coagulase-negative staphylococci, particularly those that produced a delta-like toxin, have also been associated with NEC [24]. Another possible risk factor for NEC in VLBW infants is Ureaplasma colonization of the respiratory tract, which may also suggest colonization elsewhere including the gastrointestinal tract [9]. Furthermore, after infection prevention and control measures were enhanced to manage a Staphylococcus aureus outbreak, there was a decrease in the incidence of NEC from $16 \%$ to $3 \%$ in 282 VLBW infants [25].

Although multiple studies have associated specific organisms with NEC, these organisms may represent markers of a shift in the intestinal microbiota to a dysbiotic state, and as such, an alteration of the pro- and anti-inflammatory constituents of the microbiota in the developing intestinal tract. Molecular analysis of the intestinal microbiota preceding NEC has revealed reduced overall diversity and a shift in the microbiota, often characterized by increased proportions of the Enterobacteriacea [11-13]. Previous studies have demonstrated that the pathogen recognition receptor, toll-like receptor 4 (TLR4), recognizing 
Gram-negative bacteria lipopolysaccharide, is critical for inflammation, enterocyte death, and impaired mucosal repair in animal models of NEC [26]. It has been postulated that shifts toward the Enterobacteriacea, as observed in studies of the microbiota prior to NEC, may overstimulate proinflammatory pathways [27].

Despite these reports, no single bacterial pathogen has been consistently linked with the development of NEC. However, the emergence of new sequencing techniques has allowed for detection of shifts in the premature infant intestinal microbiome, with an increase in Proteobacteria in 9 premature infants with NEC compared to 9 premature infants without NEC [12]. In our study, the organism most commonly cultured from the urine was Escherichia coli, which, along with Klebsiella pneumoniae and Enterobacter cloacae, was also one of the most common organisms implicated in a review of 17 NEC epidemics that affected both preterm and term infants [22]. In our study, Klebsiella was the most commonly isolated organism in infants who subsequently developed NEC, and Escherichia coli was the most commonly isolated organism in infants who did not develop NEC.

We found that infants with positive urine cultures for Gram-negative organisms had increased risk of NEC. In a previous study, of 478 urine cultures positive for Gram-negative organisms, 34 (7\%) were shown to have concordant blood cultures [28]. Furthermore, in infants with late-onset bloodstream infections (>48 hours after birth) caused by enteric Gram-negative bacilli, 14 of 32 infants (44\%) infected with Escherichia coli and 1 of 31 infants (3\%) infected with Klebsiella pneumoniae also had an episode of NEC [10].

A strength of this study is its large sample size derived from 322 NICUs that represented diverse patient populations. A limitation is that we included only the covariates of gestational age, inotropic support, and mechanical ventilation; there are likely other comorbidities or interventions such as human milk versus formula feeding that are associated with NEC risk which cannot be controlled for in this study [29]. Also, the non-catheterized urine specimens included in the analysis might have contributed to false-positive urine cultures. Furthermore, true UTIs could not be confirmed in the absence of additional urinary biochemical or cellular markers to complement the culture data. It is conceivable that infants 
treated with antibiotics for a false-positive non-catheterized urine culture might actually have developed NEC due to antibiotic exposure, which has been shown to increase the probability of NEC by approximately $20 \%$ per day of exposure in infants without prior sepsis [30].

In summary, we found that infants with positive urine cultures had increased risk of NEC, especially surgical NEC, and the risk was higher when limited to Gram-negative organisms. Positive urine cultures with Klebsiella were more common in infants who subsequently developed NEC, whereas positive urine cultures with Escherichia coli were more common in infants who did not develop NEC. 


\section{Funding and Conflicts of Interest}

Research reported in this publication was supported by the National Center for Advancing Translational Sciences of the National Institutes of Health under award number UL1TR001117. The content is solely the responsibility of the authors and does not necessarily represent the official views of the National Institutes of Health, which had no role in study design; the collection, analysis, and interpretation of data; the writing of the report; or the decision to submit the manuscript for publication. The first author wrote the first draft of the manuscript (no honorarium/grant/specific payment received).

Dr. Seed receives support from the National Institutes of Health and the U.S. Department of Health and Human Services (NIGMS 1R01GM108494-01), the Department of Defense (W81XWH-13-10450), The Hartwell Foundation, and the March of Dimes. Dr. Cotten receives salary support from the U.S. Department of Health and Human Services (DHHS-1R18AE000028-01). Dr. Laughon receives support from the U.S. government for his work in pediatric and neonatal clinical pharmacology (Government Contract HHSN267200700051C, PI: Benjamin, under the Best Pharmaceuticals for Children Act) and from the Eunice Kennedy Shriver National Institute of Child Health and Human Development (5K23HD068497-01). Dr. Smith receives salary support for research from the National Institutes of Health and the U.S. Department of Health and Human Services (NICHD 1K23HD060040-01 and DHHS-1R18AE000028-01); he also receives research support from industry for neonatal and pediatric drug development (www.dcri.duke.edu/research/coi.jsp). L.C.P., C.P.H., M.B., and R.H.C. have no relevant conflicts to disclose. 


\section{References}

[1] Horbar JD, Carpenter JH, Badger GJ, Kenny MJ, Soll RF, Morrow KA, et al. Mortality and neonatal morbidity among infants 501 to 1500 grams from 2000 to 2009. Pediatrics 2012;129:101926.

[2] Stoll BJ, Hansen NI, Bell EF, Shankaran S, Laptook AR, Walsh MC, et al. Neonatal outcomes of extremely preterm infants from the NICHD Neonatal Research Network. Pediatrics 2010;126:44356.

[3] Neu J, Walker WA. Necrotizing enterocolitis. N Engl J Med 2011;364:255-64.

[4] Lin PW, Nasr TR, Stoll BJ. Necrotizing enterocolitis: recent scientific advances in pathophysiology and prevention. Semin Perinatol 2008;32:70-82.

[5] Fitzgibbons SC, Ching Y, Yu D, Carpenter J, Kenny M, Weldon C, et al. Mortality of necrotizing enterocolitis expressed by birth weight categories. J Pediatr Surg 2009;44:1072-5.

[6] Hintz SR, Kendrick DE, Stoll BJ, Vohr BR, Fanaroff AA, Donovan EF, et al. Neurodevelopmental and growth outcomes of extremely low birth weight infants after necrotizing enterocolitis. Pediatrics 2005;115:696-703.

[7] Vohr BR, Wright LL, Dusick AM, Mele L, Verter J, Steichen JJ, et al. Neurodevelopmental and functional outcomes of extremely low birth weight infants in the National Institute of Child Health and Human Development Neonatal Research Network, 1993-1994. Pediatrics 2000;105:1216-26.

[8] Faustini A, Forastiere F, Giorgi Rossi P, Perucci CA. An epidemic of gastroenteritis and mild necrotizing enterocolitis in two neonatal units of a University Hospital in Rome, Italy. Epidemiol Infect 2004;132:455-65.

[9] Okogbule-Wonodi AC, Gross GW, Sun CC, Agthe AG, Xiao L, Waites KB, et al. Necrotizing enterocolitis is associated with ureaplasma colonization in preterm infants. Pediatr Res 2011;69:442-7.

[10] Cordero L, Rau R, Taylor D, Ayers LW. Enteric gram-negative bacilli bloodstream infections: 17 years' experience in a neonatal intensive care unit. Am J Infect Control 2004;32:189-95. 
[11] Morrow AL, Lagomarcino AJ, Schibler KR, Taft DH, Yu Z, Wang B, et al. Early microbial and metabolomic signatures predict later onset of necrotizing enterocolitis in preterm infants. Microbiome 2013;1:13.

[12] Mai V, Young CM, Ukhanova M, Wang X, Sun Y, Casella G, et al. Fecal microbiota in premature infants prior to necrotizing enterocolitis. PLoS One 2011;6:e20647.

[13] Wang Y, Hoenig JD, Malin KJ, Qamar S, Petrof EO, Sun J, et al. 16S rRNA gene-based analysis of fecal microbiota from preterm infants with and without necrotizing enterocolitis. ISME J 2009;3:944-54.

[14] Foglia EE, Lorch SA. Clinical predictors of urinary tract infection in the neonatal intensive care unit. J Neonatal Perinatal Med 2012;5:327-33.

[15] Bauer S, Eliakim A, Pomeranz A, Regev R, Litmanovits I, Arnon S, et al. Urinary tract infection in very low birth weight preterm infants. Pediatr Infect Dis J 2003;22:426-30.

[16] Zaidi AK, Harrell LJ, Rost JR, Reller LB. Assessment of similarity among coagulase-negative staphylococci from sequential blood cultures of neonates and children by pulsed-field gel electrophoresis. J Infect Dis 1996;174:1010-4.

[17] Stoll BJ, Hansen N, Fanaroff AA, Wright LL, Carlo WA, Ehrenkranz RA, et al. Late-onset sepsis in very low birth weight neonates: the experience of the NICHD Neonatal Research Network. Pediatrics 2002;110:285-91.

[18] Jean-Baptiste N, Benjamin DK Jr, Cohen-Wolkowiez M, Fowler VG Jr, Laughon M, Clark RH, et al. Coagulase-negative staphylococcal infections in the neonatal intensive care unit. Infect Control Hosp Epidemiol 2011;32:679-86.

[19] Munir T, Lodhi M, Hussain RM, Mubeen M. Association between periurethral colonization with uropathogens and subsequent bacteriuria in catheterized patients. J Coll Physicians Surg Pak 2009; 19:169-72. 
[20] Schlager TA, Hendley JO, Wilson RA, Simon V, Whittam TS. Correlation of periurethral bacterial flora with bacteriuria and urinary tract infection in children with neurogenic bladder receiving intermittent catheterization. Clin Infect Dis 1999;28:346-50.

[21] Korakaki E, Manoura A, Hatzidaki E, Arbiros J, Vlahakis J, Valari V, et al. Spontaneous intestinal perforation in a full-term infant: association with infection. Minerva Pediatr 2003;55:289-92.

[22] Boccia D, Stolfi I, Lana S, Moro ML. Nosocomial necrotising enterocolitis outbreaks: epidemiology and control measures. Eur J Pediatr 2001;160:385-91.

[23] de la Cochetiere MF, Piloquet H, des Robert C, Darmaun D, Galmiche JP, Roze JC. Early intestinal bacterial colonization and necrotizing enterocolitis in premature infants: the putative role of Clostridium. Pediatr Res 2004;56:366-70.

[24] Scheifele DW, Bjornson GL, Dyer RA, Dimmick JE. Delta-like toxin produced by coagulasenegative staphylococci is associated with neonatal necrotizing enterocolitis. Infect Immun $1987 ; 55: 2268-73$.

[25] Lemyre B, Xiu W, Bouali NR, Brintnell J, Janigan JA, Suh KN, et al. A decrease in the number of cases of necrotizing enterocolitis associated with the enhancement of infection prevention and control measures during a Staphylococcus aureus outbreak in a neonatal intensive care unit. Infect Control Hosp Epidemiol 2012;33:29-33.

[26] Neal MD, Sodhi CP, Dyer M, Craig BT, Good M, Jia H, et al. A critical role for TLR4 induction of autophagy in the regulation of enterocyte migration and the pathogenesis of necrotizing enterocolitis. J Immunol 2013;190:3541-51.

[27] Hunter CJ, Upperman JS, Ford HR, Camerini V. Understanding the susceptibility of the premature infant to necrotizing enterocolitis (NEC). Pediatr Res 2008;63:117-23.

[28] Downey LC, Benjamin DK Jr, Clark RH, Watt KM, Hornik CP, Laughon MM, et al. Urinary tract infection concordance with positive blood and cerebrospinal fluid cultures in the neonatal intensive care unit. J Perinatol 2013;33:302-6. 
[29] Meinzen-Derr J, Poindexter B, Wrage L, Morrow AL, Stoll B, Donovan EF. Role of human milk in extremely low birth weight infants' risk of necrotizing enterocolitis or death. J Perinatol 2009;29:57-62.

[30] Alexander VN, Northrup V, Bizzarro MJ. Antibiotic exposure in the newborn intensive care unit and the risk of necrotizing enterocolitis. J Pediatr 2011;159:392-7. 


\section{Figure legend}

Figure. Patient flow chart. GA, gestational age; BW, birth weight; GI, gastrointestinal; GU, genitourinary; NEC, necrotizing enterocolitis; DOL, day of life. 
Table 1. Demographics of infants with positive urine cultures.

\begin{tabular}{|c|c|c|c|}
\hline & $\begin{array}{c}\text { NEC } \\
\text { N=3784 }\end{array}$ & $\begin{array}{c}\text { No NEC } \\
\mathrm{N}=\mathbf{2 2 , 0 3 2}\end{array}$ & $\mathbf{P}$ \\
\hline \multirow{2}{*}{$\begin{array}{l}\text { Gestational age } \\
\text { (weeks) }\end{array}$} & & & $<0.001$ \\
\hline & & & \\
\hline$<24$ & 5 & 4 & \\
\hline $24-27$ & 55 & 51 & \\
\hline$\geq 28$ & 40 & 45 & \\
\hline Birth weight (g) & & & $<0.001$ \\
\hline$<750$ & 31 & 28 & \\
\hline $750-999$ & 33 & 30 & \\
\hline$\geq 1000$ & 36 & 42 & \\
\hline Male & 57 & 55 & 0.027 \\
\hline Inborn & 76 & 80 & $<0.001$ \\
\hline Cesarean section & 71 & 72 & 0.345 \\
\hline Race/ethnicity & & & $<0.001$ \\
\hline White & 36 & 41 & \\
\hline Black & 28 & 24 & \\
\hline Hispanic & 31 & 30 & \\
\hline Other & 5 & 5 & \\
\hline
\end{tabular}

NEC, necrotizing enterocolitis. 
Table 2. Organisms cultured from urine and risk of NEC within 7 days.

\begin{tabular}{lcc}
\hline \multicolumn{1}{c}{ Organisms } & NEC & No NEC \\
& N=334 & N=6252 \\
\hline Gram-positive cocci & & \\
CoNS & $8(2 \%)$ & $244(4 \%)$ \\
Enterococcus & $48(14 \%)$ & $869(14 \%)$ \\
Group B Streptococcus & $4(1 \%)$ & $133(2 \%)$ \\
Staphylococcus aureus & $7(2 \%)$ & $147(2 \%)$ \\
Other & $12(4 \%)$ & $179(3 \%)$ \\
Gram-negative rods & & \\
Citrobacter & $5(2 \%)$ & $128(2 \%)$ \\
Escherichia coli & $51(15 \%)$ & $1028(16 \%)$ \\
Enterobacter & $48(14 \%)$ & $717(11 \%)$ \\
Klebsiella & $62(19 \%)$ & $878(14 \%)$ \\
Proteus & $5(2 \%)$ & $128(2 \%)$ \\
Pseudomonas & $2(1 \%)$ & $177(3 \%)$ \\
Serratia & $10(3 \%)$ & $176(3 \%)$ \\
Other & $20(6 \%)$ & $222(4 \%)$ \\
Candida & $35(10 \%)$ & $808(13 \%)$
\end{tabular}

NEC, necrotizing enterocolitis; CoNS, coagulase-negative staphylococci. 
Table 3. Adjusted $^{\mathrm{a}}$ odds ratios (95\% confidence intervals) of NEC for infants with positive urine culture within 7 days prior from conditional logistic regression conditioned on postnatal age in days.

\begin{tabular}{lccc}
\hline & Medical NEC & Surgical NEC & Any NEC \\
\hline Any organism & $1.04(0.90-1.21)$ & $\mathbf{1 . 4 6}(\mathbf{1 . 1 8 - 1 . 8 1})$ & $\mathbf{1 . 1 6}(\mathbf{1 . 0 2 - 1 . 3 1})$ \\
$\begin{array}{l}\text { Gram-positive } \\
\text { organism }\end{array}$ & $0.98(0.75-1.28)$ & $1.10(0.70-1.71)$ & $1.00(0.80-1.27)$ \\
$\begin{array}{l}\text { Gram-negative } \\
\text { organism }\end{array}$ & $1.15(0.95-1.39)$ & $\mathbf{1 . 9 9}(\mathbf{1 . 5 3 - 2 . 5 9 )}$ & $\mathbf{1 . 3 7}(\mathbf{1 . 1 7 - 1 . 5 9 )}$ \\
\hline
\end{tabular}

${ }^{\mathrm{a}}$ Adjusted for gestational age and inotrope and ventilator support on the day of culture.

NEC, necrotizing enterocolitis.

Bold indicates statistically significant. 


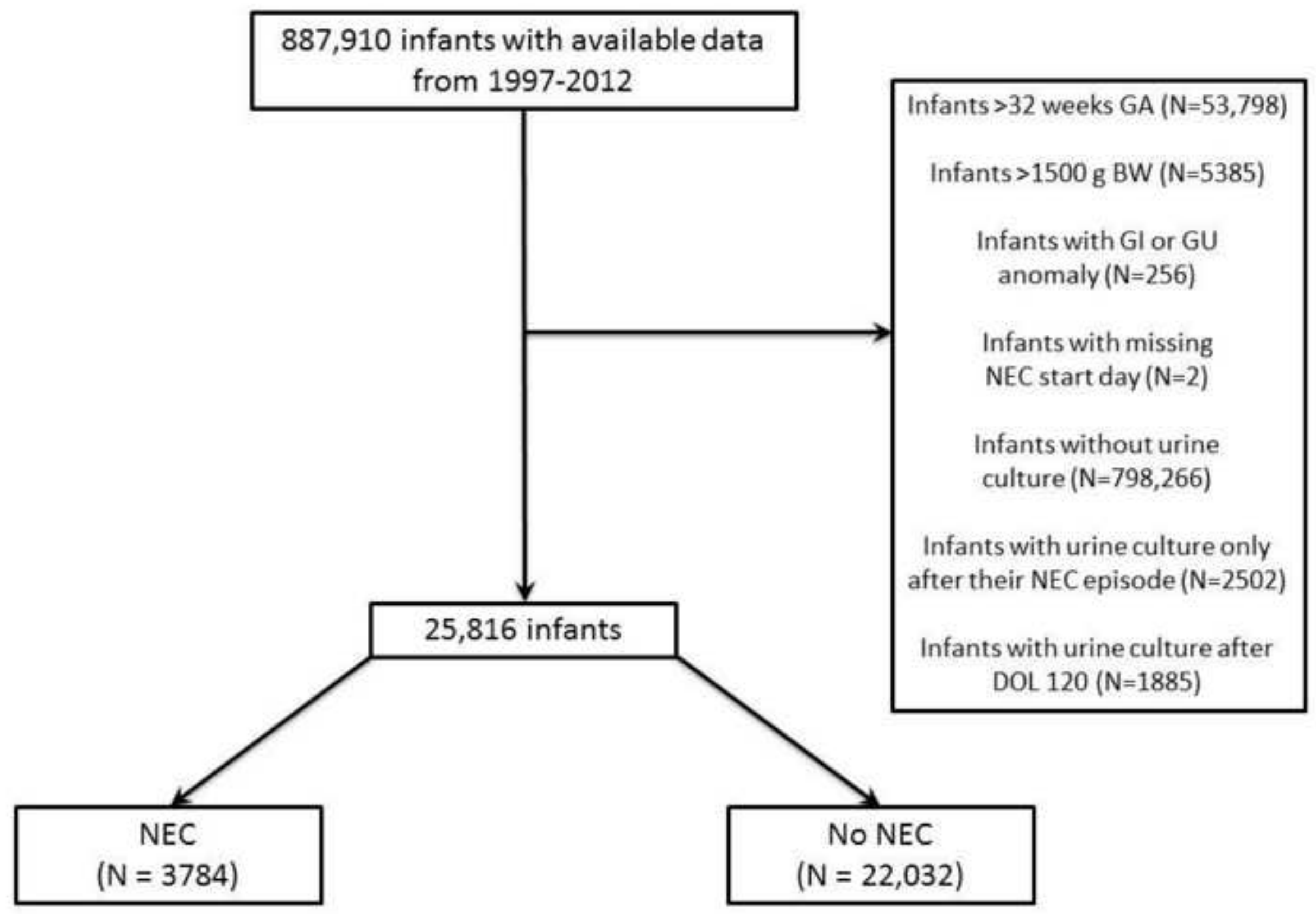

